Conclusion Urinary calprotectin had similar sensitivity and specificity for common urethral pathogens as urethral microscopy. Low calprotectin concentration correlated well with the absence of inflammation. Use of the assay is currently limited by the unknown dilution effect of urine in estimating urethral calprotectin concentrations but calprotectin is a promising biomarker of inflammation in investigating reproductive tract infections (RTI) of different aetiologies particularly where microscopy may not be available, such as in community settings.

\section{P2.114 COINFECTION OF TREPONEMA PALLIDUM AND CYTOMEGALOVIRUS (CMV): A COMPLICATED CASE OF A NEWBORN IN HUNGARY}

doi:10.1136/sextrans-2013-051184.0378

'N Mihalik, ${ }^{2} E$ Bodrogi, ${ }^{1} F$ Gódor, ${ }^{2} Z$ Nagy, ${ }^{2} \mathrm{C}$ Nádor, ${ }^{1} \mathrm{E}$ Ostorházi, ${ }^{1} \mathrm{~S}$ Kárpáti, ${ }^{1} \mathrm{M}$ Marschalkó. 'Department of Dermatology, Venerology and Dermatooncology, Budapest, Hungary; ${ }^{2}$ Perinatal Intensive Centre, ÁEK, Budapest, Hungary

Background Although congenital syphilis and congenital CMV infections are preventable they are still the major causes of perinatal mortality and morbidity. Expectant mothers from lower socioeconomical status and intravenous drug users belong to the highest risk groups for vertical transmission of infections. Here we present a coexistence of congenital syphilis and CMV infection complicated with multiplex jejunal atresia.

Methods Clinical analysis, laboratory, serological and PCR examinations.

Case presentation 990 grams, $38 \mathrm{~cm}$ height (pc.75-91) girl was born to an intravenous drug user mother on the 26th gestational week. The expectant mother did not participated in the prenatal care and early latent syphilis (RPR 1:128 positive, TPPA and TpELISA positive), genital Streptococcus agalactiae and fungal infectionwere detected shortly before delivery.

The preterm and immature girl had jaundice, oedema, gluteal haematome and petechiae. Extremely enlarged liver and spleen (reaching the hip bone) and increased muscle tone with rigid joints were found. Multiple jejunal atresia was detected by bedside X-ray examination.

Anisocytosis, thrombocytopenia, elevated liver enzymes (ASAT: 3850, ALAT: 558, GGT: 292, ALP: 436) and elevated LDH (38180) and CK (7.1) with direct hyperbilirubinaemia were found.

During microbiological examinations high copy of CMV virus number was detected by quantitative real-time PCR and syphilis serology was positive (RPR: 1:16 positive, TPPA, Tp ELISA, T. pallidum IgM immunoblot positive).

Intravenous penicilline-G (100.000 IU/ $/ \mathrm{kg} /$ dose for 10 days $)$ and intravenous ganciclovir was administered. Gancyilovir was stopped after 4 weeks because of progressing thrombocytopenia. The multiplex jejunal atresia was fixed by operation resulting in satisfactory intestinal passage.

Conclusions Although syphilis screening test within the prenatal care is mandatory in Hungary, congenital syphilis cases do occur. Immature immune system is predisposing factor for coinfections of a newborn. The symptoms of T. pallidum and CMV infection is very similar, presenting a diagnostic challenge.

\section{P2.115 GENITAL ULCERATION: INFECTION OR AUTOINFLAMMATORY DISEASE?}

doi:10.1136/sextrans-2013-051184.0379

A Geusau, A Jalili. Medical University of Vienna; 1090 Vienna, Austria

Ulcers in the genital area are usually caused by sexually transmitted infections (STIs). These include herpes, syphilis, and chancroid. However, they might also be caused by inflammatory diseases, trauma, autoimmune bullous disorders or cutaneous malignancies. In young women, Lipschütz Ulcer, or ulcus vulvae acutum, is a rare and probably underdiagnosed entity with unknown aetiology, although recent reports have associated it with the Epstein-Barr virus.

Diagnosis of most of these conditions is established by exclusion after ruling out sexually transmitted diseases, and finally with the help of histopathology. An exception is Behçet's disease (BD), a multisystem chronic inflammatory disorder, diagnosed based on the established diagnostic criteria and/or PFAPA, PAPA and TRAPS syndromes which are diagnosed by exclusion and genetic means. A complex genetic background leading to activation of the innate immune system and particularly inflammasomes through auto- or environmental antigens is presumably the causative.

Accordingly, apart from corticosteroids and colchicine, antiTNF- $\alpha$ therapy may be useful in conventional therapy-resistant refractory and severe $\mathrm{BD}$ as well in patients with PFAPA syndrome. With this abstract we would like to present and discuss our experience on diagnosis as well as treatment of "Difficult to Diagnosis and Treat" patients with genital ulcers from the DIAID of the Department of Dermatology at the Medical University of Vienna.

Beside STIs as the cause genital ulcers, autoinflammatory disorders should be also considered. Here, novel approaches such as antiTNF-a and IL-1R blockers are promising therapeutic strategies and warrant multicenter clinical studies.

\section{P2.116 GENITAL ULCER, NOT ALWAYS A CLASSIC SEXUALLY TRANSMITTED DISEASE: CASE REPORT OF VULVAR TUBERCULOSIS}

doi:10.1136/sextrans-2013-051184.0380

${ }^{1}$ E S Cordeiro, ${ }^{1} \mathrm{~A}$ C Pontes, ${ }^{1 P}$ C Giraldo, ${ }^{2} \mathrm{M}$ R L Passos, 'R L G do Amaral. 'State University of Campinas, Campinas, Brazil; ${ }^{2}$ Fluminense Federal University, Niterói, Brazil

Background Tuberculosis is a very common disease worldwide. In 2010 there were 71000 cases reported in Brazil. The genital presentation has a prevalence of 8-10 million cases worldwide. The vulvar/ vaginal involvement is less than $2 \%$ of the cases. The clinical presentation can be variable and genital ulcers(GU) can be confused with sexually transmitted diseases(STD) such as syphilis and chancroid. Methods/results(case report) MJS, 73y, G9P9, rural worker, attended at a clinic specialised in genital infections at the State University of Campinas-(UNICAMP)-Brazil with dysuria and pain/ burning in the vulva for 3 months. Physical examination revealed ulceration of $3 \mathrm{~cm}$ in small genital right lip with bilateral inguinal painless adenopathy. The ulcer biopsy showed chronic granulomatous inflammation and search for AFB and fungi by techniques of Gomori and Ziehl-Nielssen were negative. Vaginal bacterioscopy and serologies were regular. Vaginal wall biopsy with search and culture resulted positive for complex M.tuberculosis. The Mantoux test resulted in strong reaction- $13 \mathrm{~mm}$ and the AFB sputum (3samples) was negative. Chest radiography showed no abnormalities. It was introduced the treatment with isoniazid+rifampicin for 6 months. After 60 days the pacient presented ulcer resolution.

Conclusion The authors describe an unusual presentation of the disease, a painless chronic ulcer, for which differential diagnosis of GU by STD should be clarified. The isolation of M.tuberculosis in the culture of the ulcer is the gold standard for the diagnosis of genital tuberculosis. The ulcers caused by herpes virus, syphilis or chancroid are common, but it is essential to think of an infection by M.tuberculosis, especially in countries where the prevalence of the disease is high. It was suggested that the sputum of a subject with pulmonary tuberculosis when used as a lubricant during intercourse can transmit genital disease, making it an eventual STD. Chronic GU should be biopsied and the possibility of unusual etiologies should be considered. 